New windows on massive stars: asteroseismology, interferometry, and spectropolarimetry

Proceedings IAU Symposium No. 307, 2014

G. Meynet, C. Georgy, J. H. Groh 8 Ph. Stee, eds.

(C) International Astronomical Union 2015 doi:10.1017/S1743921314006917

\title{
Zooming into Eta Carinae with interferometry
}

\author{
Jose H. Groh \\ Geneva Observatory, Geneva University, Chemin des Maillettes 51, CH-1290 Sauverny, \\ Switzerland \\ email: jose.groh@unige.ch
}

\begin{abstract}
Shaped by strong mass loss, rapid rotation, and/or the presence of a close companion, the circumstellar environment around the most massive stars is complex and anything but spherical. Here we provide a brief overview of the high spatial resolution observations of Eta Carinae performed with the Very Large Telescope Interferometer (VLTI). Special emphasis is given to discuss VLTI/AMBER and VLTI/VINCI observations, which directly resolve spatial scales comparable to those where mass loss originates. Studying scales as small as a few milliarcseconds allows us to investigate kinematical effects of rotation and binarity in more detail than ever before.
\end{abstract}

Keywords. stars: atmospheres stars: individual (Eta Carinae) stars: mass-loss stars: rotation stars: variables: general supergiants

Recent advances in long-baseline optical interferometry allow one to directly resolve astrophysical objects at the milli-arcsecond (mas) spatial scale. For Galactic massive stars, this approaches scales comparable to those where mass loss originates, stellar surfaces and winds become distorted by rotation, and the presence of close companions can be directly diagnosed. Mass-loss rates generally increase as evolution proceeds, causing the outer hydrostatic layers of massive stars to eventually become hidden by an opticallythick stellar wind. Thus, as massive stars evolve, their appearance is increasingly affected by mass loss - a phenomenon that can be directly probed with optical interferometry. In addition, the interplay between mass loss and rotation will also shape the circumstellar environment around massive stars in a complex manner (Owocki et al. 1996). Likewise, if a massive companion star is present, strong disturbances in the stellar wind structure may occur, in particular if the wind of the companion has enough momentum to cause the presence of a wind-wind collision zone between the two stars (Stevens et al. 1992).

Because of its large brightness and unique nature among massive stars, Eta Carinae has been a natural target for optical interferometers. Most, if not all groups working on Eta Car, support a massive binary scenario with an eccentric orbit, as suggested by Damineli et al. (1997). The orbital period is 2022.7 $\pm 1.3 \mathrm{~d}$ (Damineli et al. 2008b), and the eccentricity $e \geqslant 0.9$ (Corcoran 2005). The orbital orientation has been controversial (see discussion in Madura et al. 2012), but the first determination in 3-D space suggests an orbital inclination angle $i \approx 130^{\circ}$ to $145^{\circ}$, longitude of periastron $\omega \approx 240^{\circ}$ to $285^{\circ}$, and an orbital axis projected on the sky at a position angle $\mathrm{PA}_{z} \approx 302^{\circ}$ to $327^{\circ}$ from North to East (Madura et al. 2012). The combined mass of the system is larger than 110 solar masses (Hillier et al. 2001), and only loose constraints on the individual masses exist. The combined luminosity is $\simeq 5 \cdot 10^{6} L_{\odot}$ (Davidson \& Humphreys 1997), and thought to be accounted for mainly by the primary star (hereafter $\eta_{\mathrm{A}}$ ), since it dominates the ultraviolet, optical, and near-infrared spectrum (Hillier et al. 2001, 2006; Groh et al. 2012, hereafter G12). The most recent spectroscopic analysis suggests that $\eta_{\mathrm{A}}$ has an effective 
temperature of $\sim 9,400 \mathrm{~K}$, mass-loss rate of $8.5 \cdot 10^{-4} M_{\odot} \mathrm{yr}^{-1}$, and wind terminal velocity of $\sim 420 \mathrm{~km} \mathrm{~s}^{-1}$ (G12; see also Hillier et al. 2001, 2006). Concerning the companion star (hereafter $\left.\eta_{\mathrm{B}}\right)$, only indirect constraints exist on its temperature $\left(T_{\text {eff }} \simeq 36,000-\right.$ 41,000 K, Mehner et al. 2010; see also Verner et al. 2005; Teodoro et al. 2008) and luminosity $\left(10^{5} L_{\odot} \leqslant L_{\star} \leqslant 10^{6} L_{\odot} ;\right.$ Mehner et al. 2010). These come from ionization studies of the ejecta surrounding Eta Car, as $\eta_{\mathrm{B}}$ has never been observed directly. Xray studies have constrained the wind properties of $\eta_{\mathrm{B}}\left(v_{\infty} \sim 3000 \mathrm{~km} \mathrm{~s}^{-1}\right.$ and $\dot{M} \sim$ $1.4 \cdot 10^{-5} M_{\odot} \mathrm{yr}^{-1}$; e.g., Parkin et al. 2011).

\section{A close view of Eta Carinae with the VLTI}

Here we review Eta Car VLTI observations obtained in the K-band with the beamcombiner instruments VINCI and AMBER. VINCI (now decommissioned) operated as a broadband instrument and combined light from 2 telescopes delivering visibilities. These are the amplitude of the Fourier transform of an object's brightness distribution on the sky, and visibilities are directly related to sizes. In contrast to VINCI, AMBER combines light from up to 3 telescopes and has 3 spectral resolution modes: low- (LR, $R \sim 30$ ), medium- (MR, $R \sim 1500$ ), and high-resolution (HR, $R \sim 12000$ ). In addition to visibilities, AMBER delivers differential phases, which are related to photocenter shifts, and closure phases, which measures how point-symmetric the brightness distribution is.

Eta Carinae was observed with VINCI from 2001 November until 2004 January, using mainly the siderostats. These dataset have been discussed in van Boekel et al. (2003) and Kervella (2007). AMBER observations with the UTs in MR and HR, obtained between 2004-2005, were reported by Weigelt et al. (2007).

VINCI provided our first milli-arcsecond view of Eta Car (van Boekel et al. 2003) and two main breakthroughs: the K-band (pseudo) photosphere was spatially resolved and the brightness distribution was non-spherical in 2003. van Boekel et al. (2003) reported visibility measurements of Eta Car for a range of baseline position angles (P.A.) on the sky. These data were analyzed by these authors assuming that the brightness distribution of Eta Car can be represented by a two-dimensional Gaussian. Under this assumption, the VINCI dataset can be fitted by an ellipsoid with a ratio of major to minor axis of $1.25 \pm 0.05$, with the major axis aligned at P.A. $=134 \pm 7^{\circ}$ East of North. The inferred Gaussian full-width at half maximum (FWHM) diameters are between $\sim 6-8.5$ mas depending on P.A. This elongation of the K-band emission has been explained as being due to the presence of a dense polar wind generated by the rapid rotation of $\eta_{\mathrm{A}}$ (van Boekel et al. 2003). However, any influence from $\eta_{\mathrm{B}}$ was not considered at this point, which is worth mentioning given that the 2003 VINCI data were obtained at orbital phase $\phi=10.93$, i.e. relatively close to periastron passage of $\eta_{\mathrm{B}}$. Here we assume the ephemeris from Damineli et al. (2008a), the orbital cycle labeling from Groh \& Damineli (2004), and that periastron occurs at $\phi=0$.

AMBER revolutionized again our view of Eta Car, as it became possible to resolve the object within spectral lines and, thus, obtain kinematical information. Weigelt et al. (2007) were able to resolve Eta Car in multiple spectral channels within Bracket $\gamma$, HeI $2.058 \mu \mathrm{m}$, and K-band continuum, finding $50 \%$ encircled-energy diameters of 9.6, 6.5, and 4.2 mas, respectively. The different values of the K-band continuum diameters found with VINCI and AMBER are consistent when accounting for the fact that a Gaussian approximation is not adequate for the brightness distribution of Eta Car (Weigelt et al. 2007; Kervella 2007). The AMBER dataset also supports a similar elongation of the K-band emission along P.A. $=120 \pm 15^{\circ}$. The Hillier et al. (2001) model reproduces the K-band continuum visibilities, indicating that the dense stellar wind of $\eta_{\mathrm{A}}$ is indeed being 
resolved with AMBER. At least during 2004-2005, it does not seem that a significant amount of hot dust is present within the field-of-view of the UTs ( $\sim 70$ mas $)$. The extension of the $\operatorname{Br} \gamma$ emitting region is also roughly reproduced by the spherical Hillier et al. (2001) model.

The AMBER data shows significant signals in the differential and closure phases within the $\mathrm{Br} \gamma$ and HeI $2.058 \mu \mathrm{m}$ lines. These quantities reveal a complex pattern of photocenter shifts and image asymmetries as a function of velocity within the line. The $\mathrm{Br} \gamma$ behavior has been interpreted in the context of a dense polar wind with the rotation axis tilted at $\sim 41^{\circ}$ from the observer. A geometrical model has been developed by Weigelt et al. (2007) to explain the relative photocenter shifts seen in the blue and red wings of $\operatorname{Br} \gamma$.

AMBER also shed light on the binarity of Eta Car, although light from $\eta_{\mathrm{B}}$ was not detected. A lower limit for the K-band brightness ratio of $\eta_{\mathrm{A}}$ to $\eta_{\mathrm{B}}$ of $\sim 50$ has been determined (Weigelt et al. 2007), which is consistent with the brightness ratio of 200 expected for an O star companion with $T_{\text {eff }} \simeq 34,000 \mathrm{~K}$ and $L_{\star}=10^{6} L_{\odot}$ (Hillier et al. 2006). Given the findings of Mehner et al. (2010) that $L_{\star}=10^{6} L_{\odot}$ is likely an upper limit, and that $\eta_{\mathrm{B}}$ could have $L_{\star}$ as low as $10^{5} L_{\odot}$, it may well be that one needs to be sensitive to brightness ratios of 500 or higher to directly detect $\eta_{\mathrm{B}}$.

\section{An elongated K-band photosphere: rotation or binary induced?}

Given the ubiquitous evidence for binarity in Eta Car, one may wonder how much the presence of $\eta_{\mathrm{B}}$ would affect the interferometric observables described above, and the conclusion that a rapidly rotating $\eta_{\mathrm{A}}$ causes the deformation in the K-band photosphere. With the goal of constraining the rotational velocity $\left(v_{\text {rot }}\right)$ of $\eta_{\mathrm{A}}$ and probing the influence of $\eta_{\mathrm{B}}$ on the wind of $\eta_{\mathrm{A}}$, Groh et al. (2010) analyzed the aforementioned VINCI and AMBER interferometric measurements of Eta Car with 2D radiative transfer models based on the Busche \& Hillier (2005) code.

Ignoring the presence of $\eta_{\mathrm{B}}$, rotation and the spatial orientation of the rotation axis of $\eta_{\mathrm{A}}$ can be investigated based on the effects of rotation on the wind density structure, which determines the geometry of the $K$-band emitting region. Prolate wind models with a ratio of $v_{\text {rot }}$ to the critical velocity for break-up $\left(v_{\text {crit }}\right)$ of $W=0.77-0.92$ (assuming the prescription from Owocki et al. 1996), inclination angle of the rotation axis of $i=60^{\circ}-90^{\circ}$, and position angle on the sky of P.A. $=108^{\circ}-142^{\circ}$ reproduce
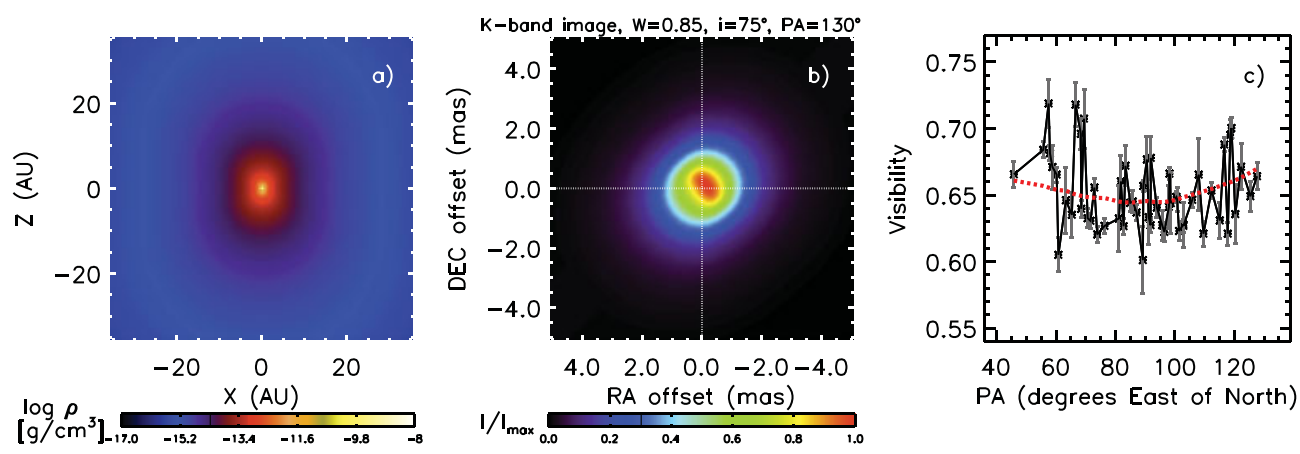

Figure 1. (a): Density structure of the latitude-dependent wind models of $\eta_{\mathrm{A}}$ in the x-z plane (i. e., equator-on). (b): K-band image projected on the sky. (c): VINCI visibilities for the $24 \mathrm{~m}$ baseline as a function of baseline P.A. (connected black asterisks) compared to the respective model prediction (red dotted line). This model assumes a prolate wind with $W=0.85, i=75^{\circ}$, P.A. $=130^{\circ}$. Adapted from Groh et al. (2010). 
simultaneously K-band continuum visibilities from VLTI/VINCI and closure phase measurements with VLTI/AMBER (see Fig. 1). Interestingly, oblate models with $W=$ $0.73-0.90$ and $i=80^{\circ}-90^{\circ}$ produce similar fits to the interferometric data, but require P.A. $=210^{\circ}-230^{\circ}$. Therefore, both prolate and oblate models suggest that the rotation axis of the primary star is not aligned with the Homunculus polar axis. While a prolate wind is thought to arise in a gravity-darkened, fast-rotating star with a radiative envelope (Owocki et al. 1996, 1998), an oblate wind can be produced by a fast-rotating star when gravity-darkening is not important (Bjorkman \& Cassinelli 1993; Owocki et al. 1994, 1996, 1998).

$\eta_{\mathrm{A}}$ has been routinely referred to as the prototype of a massive star with a fast, dense polar wind created by rapid stellar rotation, even though the system is believed to contain a massive companion, $\eta_{\mathrm{B}}$. What happens when the influence of $\eta_{\mathrm{B}}$ is taken into account? Three-dimensional hydrodynamical simulations have shown that the geometry of the wind of $\eta_{\mathrm{A}}$ is severely affected by the wind of $\eta_{\mathrm{B}}$, which creates a low-density WWC cavity in the wind of $\eta_{\mathrm{A}}$ and a thin, dense wind-wind interacting region between the two winds (Pittard \& Corcoran 2002; Okazaki et al. 2008; Parkin et al. 2009, 2011; Madura et al. 2012). Groh et al. (2010) presented an extension of the $2 \mathrm{D}$ radiative transfer code of Busche \& Hillier (2005) to handle massive binary systems (see also Groh et al. 2012 for further details on the implementation). Interestingly, when the aforementioned density effects arising due to the presence of $\eta_{\mathrm{B}}$ are taken into account, an otherwise spherical primary wind carved by the companion can explain the observed VINCI and AMBER data (Groh et al. 2010). Figure 2a presents the assumed density structure of $\eta_{\mathrm{A}}$ 's carved wind, with a distance of the apex of the WWC to $\eta_{\mathrm{A}}$ of $d_{\mathrm{apex}}=10 \mathrm{AU}$, half-opening angle of the cavity of $\alpha=54^{\circ}$, and width of the shocked walls of $\delta \alpha=3^{\circ}$. The best-fit orbital orientation is sketched in Fig. $2 \mathrm{~b}$, with $i=41^{\circ}, \omega=270^{\circ}$, and P.A. $=40^{\circ}$. Note that a similar good fit (Fig. 2d) would be also obtained with the orbital inclination suggested by Madura et al. $(2012)\left(i=138^{\circ}\right)$.

Therefore, assuming the standard orbital and wind parameters of Eta Car, even if $\eta_{\mathrm{A}}$ has a spherical wind, its inner density structure can be sufficiently disturbed by $\eta_{\mathrm{B}}$, mimicking the effects of a prolate/oblate latitude-dependent wind in the available interferometric observables in the K-band continuum. Therefore, fast rotation may not be the only explanation for the interferometric observations (Groh et al. 2010).

We have presented a brief overview of the high spatial resolution investigations of the Eta Car massive binary system reported in the literature. We hope the reader is
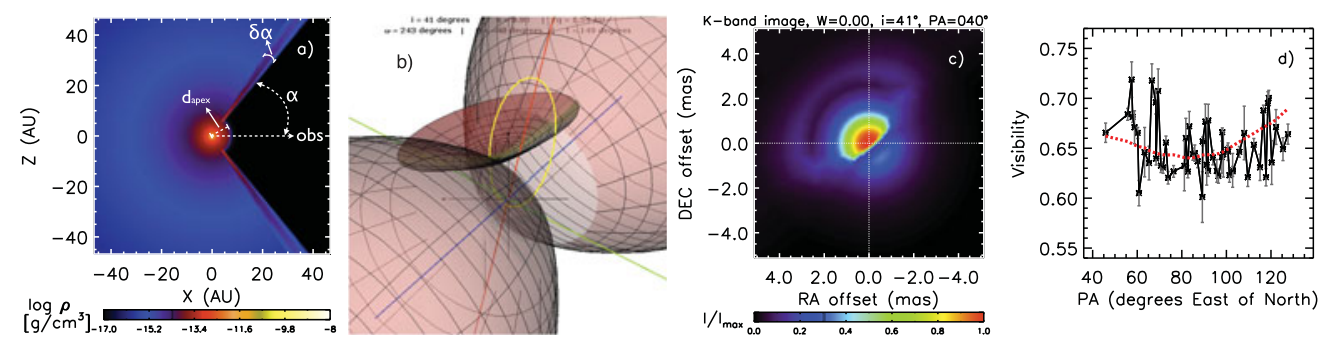

Figure 2. Panels a,c,d: Similar to Figure 1, but for the $\eta_{\mathrm{A}}$ model with a spherical wind including a cavity and compressed walls created by the wind of $\eta_{\mathrm{B}}$. The model is appropriate for the VINCI observations $(\phi=10.93)$. See text for model parameters. Panel $b$ : Sketch of the binary orbit (yellow) and the shock cone orientation at $\phi=10.93$ relative to the Homunculus (not to scale), assuming a counterclockwise motion of $\eta_{\mathrm{B}}$ on the sky. The orbital plane is assumed to be in the skirt plane with the orbital axis (blue) aligned to the Homunculus axis of symmetry. North is up and East is to the left. From Groh et al. (2010). 
convinced that zooming into Eta Car significantly increases the amount of information available and the constraints that can be put on hydrodynamic and radiative transfer models. The joint effort of many observers and theoreticians have shown that the binary interaction in Eta Car is complex, and no firm constraints on the individual masses exist yet. We encourage efforts into this direction, since this may present a rare opportunity to weight one of the most massive stars in the Galaxy.

\section{Acknowledgements}

I would like to thank my collaborators in studies of high spatial resolution observations of Eta Car: T. Madura, T. R. Gull, S. P. Owocki, D. J. Hillier, G. Weigelt, O. Absil, J.-P. Berger, H. Sana, J.-B. Le Bouquin, and M. de Becker.

\section{References}

Bjorkman, J. E. \& Cassinelli, J. P. 1993, ApJ 409, 429

Busche, J. R. \& Hillier, D. J. 2005, AJ 129, 454

Corcoran, M. F. 2005, AJ 129, 2018

Damineli, A., Conti, P. S., \& Lopes, D. F. 1997, New. Astron. 2, 107

Damineli, A., Hillier, D. J., Corcoran, M. F., et al. 2008a, MNRAS 386, 2330

Damineli, A., Hillier, D. J., Corcoran, M. F., et al. 2008b, MNRAS 384, 1649

Davidson, K. \& Humphreys, R. M. 1997, ARA\& A 35, 1

Groh, J. H. \& Damineli, A. 2004, Information Bulletin on Variable Stars 5492, 1

Groh, J. H., Hillier, D. J., Madura, T. I., \& Weigelt, G. 2012, MNRAS 423, 1623

Groh, J. H., Madura, T. I., Owocki, S. P., Hillier, D. J., \& Weigelt, G. 2010, ApJ (Letters) 716, L223

Hillier, D. J., Davidson, K., Ishibashi, K., \& Gull, T. 2001, ApJ 553, 837

Hillier, D. J., Gull, T., Nielsen, K., et al. 2006, ApJ 642, 1098

Kervella, P. 2007, A\&A 464, 1045

Madura, T. I., Gull, T. R., Owocki, S. P., et al. 2012, MNRAS 420, 2064

Mehner, A., Davidson, K., Ferland, G. J., \& Humphreys, R. M. 2010, ApJ 710, 729

Okazaki, A. T., Owocki, S. P., Russell, C. M. P., \& Corcoran, M. F. 2008, MNRAS 388, L39

Owocki, S. P., Cranmer, S. R., \& Blondin, J. M. 1994, ApJ 424, 887

Owocki, S. P., Cranmer, S. R., \& Gayley, K. G. 1996, ApJ (Letters) 472, L115

Owocki, S. P., Cranmer, S. R., \& Gayley, K. G. 1998, Ap\&SSS 260, 149

Parkin, E. R., Pittard, J. M., Corcoran, M. F., \& Hamaguchi, K. 2011, ApJ 726, 105

Parkin, E. R., Pittard, J. M., Corcoran, M. F., Hamaguchi, K., \& Stevens, I. R. 2009, MNRAS 394,1758

Pittard, J. M. \& Corcoran, M. F. 2002, A\&A 383, 636

Stevens, I. R., Blondin, J. M., \& Pollock, A. M. T. 1992, ApJ 386, 265

Teodoro, M., Damineli, A., Sharp, R. G., Groh, J. H., \& Barbosa, C. L. 2008, MNRAS 387, 564 van Boekel, R., Kervella, P., Schöller, M., et al. 2003, A\&A 410, L37

Verner, E., Bruhweiler, F., \& Gull, T. 2005, ApJ 624, 973

Weigelt, G., Kraus, S., Driebe, T., et al. 2007, A\& A 464, 87

\section{Discussion}

Puls: Two comments. 1. When transforming the wind shape to the rotational speed, one has to be careful, because a direct relation is only provided if the ionization structure does not change from pole to equator. 2. For such high mass-loss rates, also optically-thick clumping effects (porosity and vorosity) might need to be considered.

GROH: I totally agree with your remarks. We used the Owocki et al. parametrization that is indeed more appropriate for $\mathrm{O}$ stars. However, regardless of the relationship used, 
the strong deformation of the K-band photosphere points out to a relatively high ratio of rotational over critical speed.

DE Koter: You find that the axis of symmetry of the Homunculus is offset from the rotational axis of the star (though they are aligned as found by van Boekel et al. 2003). Do you have ideas as to what may have caused this?

Gron: Great question. Van Boekel et al. assumed that the rotational axis and the Homunculus symmetry axes were aligned, since they could only measure the azimuthal angle (i.e. the position angle on the sky), but not the inclination angle. Our results suggest that the inclination angle is offset by at least 20 degrees. One possibility is that the angular momentum loss was not axisymmetric during the formation of the Homunculus, which would have caused a recoil of the rotational axis of the star. Another possibility is that the secondary star ejected the Homunculus, so the Homunculus symmetry axis would be related to the rotational axis of the secondary star, and not that of the primary which is what we measure.

WeIs: I have a comment to the previous question by A. de Koter. You also have to create an equatorial plane/disk. My question: you showed a spheroidal structure in the K-band from Eta Car that represents the shape and reveals the rotation and inclination of the rotational axis. It is however known that LBVs in their cool phases (which can be multiple) do inflate their radius and form pseudo-photospheres, mimicking a cool star. Do the K-band observation probe this pseudo-photosphere or can we look into the deeper layers?

GROH: Unfortunately we cannot look deeper that the surface where the optical depth is roughly $2 / 3$, so the K-band observations probe the pseudo-photosphere at this wavelength. This is a physical limit, as we can only observe the surface where the photons are thermalized and finally decouple from the matter.

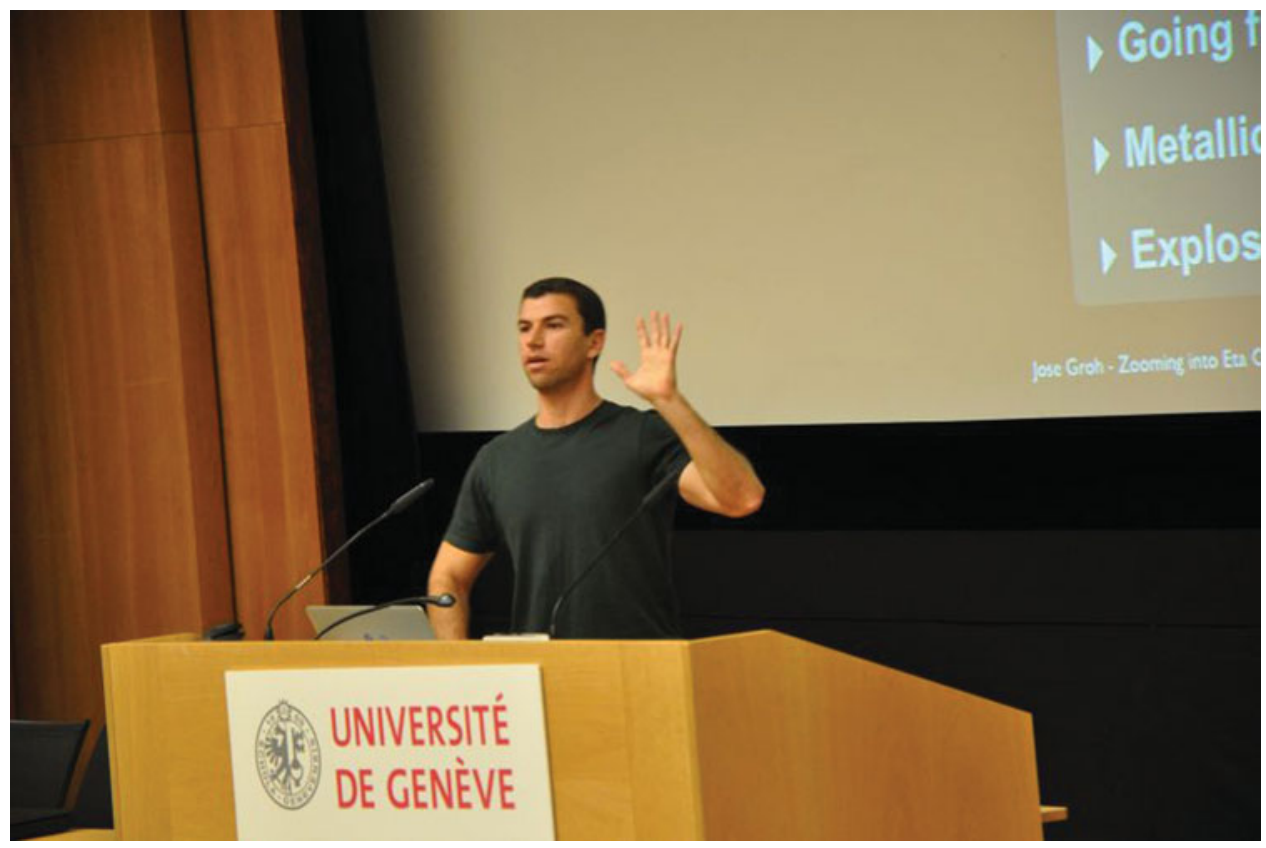

José Groh 